\title{
Commentary: Near-infrared technology continues to evolve, but the holy grail remains elusive
}

\author{
Richard A. Jonas, MD
}

From the Children's National Heart Institute, Children's National Medical Center, Washington, DC.

Disclosures: Author has nothing to disclose with regard to commercial support.

Received for publication Aug 5, 2019; accepted for publication Aug 5, 2019; available ahead of print Oct 17, 2019

Address for reprints: Richard A. Jonas, MD, Cardiac Surgery, Children's National Heart Institute, Children's National Medical Center, 111 Michigan Ave NW, Washington, DC 20010 (E-mail: rjonas@ childrensnational.org). J Thorac Cardiovasc Surg 2020;159:2026-7

$0022-5223 / \$ 36.00$

Copyright $(2019$ by The American Association for Thoracic Surgery

https://doi.org/10.1016/j.jtcvs.2019.08.046

The article by Cheng and colleagues ${ }^{1}$ in this issue of the Journal reflects the ongoing development and increasing sophistication of noninvasive optical methods for determining cerebral blood flow and oxygenation status of the neonatal and infant brains. As fiberoptic technology was rapidly evolving in the 1970s and 1980s, Frans Jobsis at Duke pioneered the clinical application of near-infrared light to determine oxygenation status of organs, including the brain. The fact that the neonatal skull is virtually transparent to near-infrared light makes the neonate and young infant particularly suitable for near-infrared spectroscopic monitoring. With a near-infrared light transmitter and receiver system, and with inferences regarding the pathlength traversed by the directly transmitted as well as the reflected light beam, it is possible to determine the oxygenation status of the blood within the skull and by inference of the brain by using relative levels of oxyhemoglobin and deoxyhemoglobin. Jobsis also strove to measure a much more subtle index, the redox status of cytochrome $\mathrm{a}, \mathrm{a}_{3}$, the terminal cytochrome of the intracellular electron transport chain. Since the early work of Jobsis, a number of academic and commercial groups have developed increasingly sophisticated methods, including the system applied in the study of Cheng and colleagues, ${ }^{1}$ which uses frequency domain near-infrared spectroscopy. This instrument uses multiple infrared transmitters and detectors and multiple frequencies within the near-infrared spectrum. This combination allows more accurate determination of path length and thus more accurate determinations of oxygenation status. The other technique that Cheng and colleagues ${ }^{1}$ have applied is diffusion correlation spectroscopy, which is a variation on laser Doppler flowmetry. By observing the Doppler shift in frequency of a nearinfrared laser beam, inferences can be made regarding the blood flow within the interrogated volume of brain tissue. The instrument that Cheng and colleagues ${ }^{1}$ have used in this study is the result of ongoing work from the laboratory at the University of Pennsylvania of Britton Chance, who was also a pioneer in the early development of near-

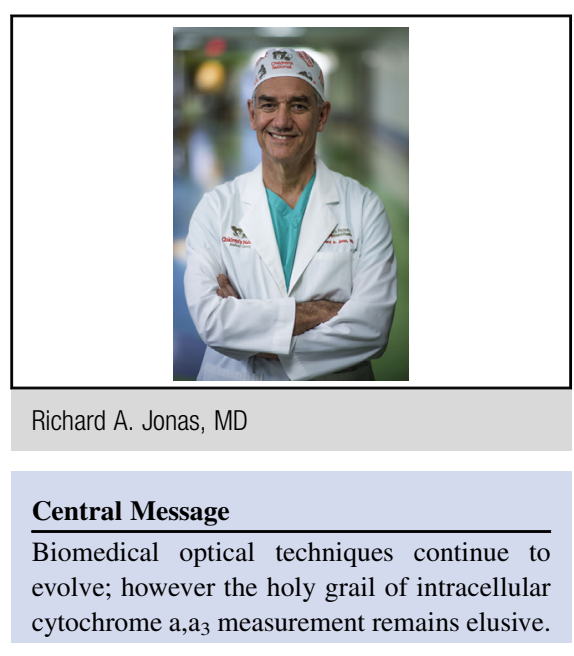

See Article page 2012. infrared spectroscopy. A small number of validation studies of diffusion correlation spectroscopy have been encouraging; however, there is little doubt that instruments for noninvasive assessment of cerebral oxygenation and cerebral blood flow will continue to evolve. It is to be hoped that the holy grail of near-infrared spectroscopy, which was a driving force behind the early work of Jobsis, will allow intracellular redox status to be determined by accurate measurement of cytochrome $a, a_{3}$, a hope that until now has not been realized. ${ }^{2}$

Cheng and colleagues ${ }^{1}$ have applied their dual nearinfrared technology to 3 groups of neonates with congenital heart disease. Their graphical abstract nicely captures the central findings of the study and perhaps for most readers provides few surprises. Transposition of the great arteries, with its attendant reduced arterial oxygen saturation, is associated with increased oxygen extraction in the brain before reparative surgery. After surgery, the brain's oxygenation status increases and the oxygen extraction decreases, presumably as a direct consequence of increased arterial oxygen saturation and therefore increased oxygen delivery. Patients with single-ventricle circulations, who all have a steal from the systemic into the pulmonary circulation, have reduced cerebral blood flow, reduced cerebral oxygenation, and increased oxygen extraction preoperatively. The reduced blood flow is relieved by the first-stage operation, regardless of whether a Sano shunt or a Blalock shunt is placed; however, deoxygenation of the brain persists after 
surgery, no doubt as a consequence of reduced arterial oxygen saturation reflecting the mixed single-ventricle physiology.

Although it may be reassuring to find that our expectations regarding these findings are confirmed by the dual infrared technology of Cheng and colleagues, ${ }^{1}$ it is important to recognize that this technology, and in fact all current near-infrared spectroscopic technology, is not measuring intracellular oxygenation status. It is telling us exactly the same information that we can derive if we know the arterial oxygen saturation of blood flowing to the brain and the mixed venous oxygen saturation of the blood that is coming from the brain. The latter can be obtained by placing a continuous oxygen saturation probe in the jugular venous bulb at the top of the internal jugular vein as blood exits the cranium. Whether directly measured or indirectly measured by spectroscopy, the oxygenation status of hemoglobin does not tell us whether the oxygen that is being supplied to the brain is getting to where it needs to go ie, the intracellular electron transport chain. Major shifts occur in oxyhemoglobin dissociation around the time of surgery related to changes in temperature, $\mathrm{pH}$, and 2,3-diphosphoglycerate levels. We thus cannot safely make the assumption that all brain cells are receiving the oxygen that they require simply because there appears to be adequate arterial oxygenation and reasonable venous oxygen saturation. Under normal physiologic circumstances, this is not an unreasonable assumption; however, the many changes that occur in circulatory physiology during cardiac surgery and circulatory arrest invalidate that assumption. Until the holy grail of continuous noninvasive real-time monitoring of the cytochrome electron transport chain is available, we are still flying in the dark when it comes to monitoring oxygen status of the brain during cardiac surgery.

\section{References}

1. Cheng HH, Ferradal SL, Vyas R, Wigmore D, McDavitt E, Soul JS, et al. Abnor malities in cerebral hemodynamics and changes with surgical intervention in neonates with congenital heart disease. J Thorac Cardiovasc Surg. 2020;159: 2012-21.

2. Sakamoto T, Jonas RA, Stock UA, Hatsuoka S, Cope M, Springett RJ, et al. Utility and limitations of near-infrared spectroscopy during cardiopulmonary bypass in a piglet model. Pediatr Res. 2001;49:770-6. 